\title{
ON DUAL INTEGRAL EQUATIONS ARISING IN PROBLEMS OF BENDING OF ANISOTROPIC PLATES
}

\author{
B. D. AGgARWALA and C. NASIM \\ Department of Mathematics and Statistics \\ University of Calgary \\ Calgary, Alberta, Canada \\ T2N 1N4 \\ (Received March 6, 1991 and in revised form Apri1 11, 1991)
}

\begin{abstract}
In this paper we consider dual integral equations, which arise in boundary value problems of bending of anisotropic plates. The function involved in these equations is a linear combination of elementary function, which turns out to be a particular case of a class of Fourier kernels, [2]. The method used here for solving the equations is some what similar to the method used for solving dual integral equations of Titchmarsh type, [1].
\end{abstract}

KEY WORDS AND PHRASES. Dual integral equations, Bessel functions, Erdelyi-Kober operators, Parseval theorem, Mellin transforms.

1991 AMS SUBJECT CLASSIFICATION CODE: 45F10.

\section{INTRODUCTION}

In this note we consider dual integral equations

$$
\begin{aligned}
& \int_{0}^{\infty} \phi(t) h_{1}(x t) d t=f(x), \quad 0<x<1 \\
& \int_{0}^{\infty} \phi(t) h_{2}(x t) d t=g(x), \quad x>1,
\end{aligned}
$$

where $\phi$ is the unknown function and

$$
\begin{aligned}
& h_{1}(x)=e^{-x} \mp \cos x \pm \sin x \\
& h_{2}(x)=x^{\nu} h_{1}(x), \quad-1 \leq \nu \leq 1 .
\end{aligned}
$$

The method we employ for solving the system (1.1) is similar to a method developed by Nasim \& Sneddon, [1]. This procedure has been very effective for solving dual integral equations when the functions $h_{1}$ and $h_{2}$ involve Bessel functions $J_{v}, Y_{v}$ and $K_{v}$. As in almost all the papers concerned with dual integral equations, here also, the solutions are not derived in a rigorous fashion. But that is not to say that the analysis in this paper cannot be made rigorous by imposing appropriate conditions on the functions involved.

It is worth noting that in our case the functions $h_{1}$ are a special case of Fourier kernels defined by us elsewhere, [2]. Also we wish to point out that the functions $h_{1}(x)$ 
satisfy the differential equation

$$
\frac{\mathrm{d}^{4}}{\mathrm{dx}} \mathrm{h}(\lambda \mathrm{x})=\hat{\lambda} \mathrm{h}(\lambda \mathrm{x})
$$

and equations (1.1) would therefore arise in many situations where we wanted to solve a partial Differential Equation involving the operator $\frac{\partial^{4}}{\partial x^{4}}$ A particular such case e.g., is the bending of an anisotropic plate where the lateral deflection $\omega$ satisfies the equation

$$
\frac{\partial^{4} \omega}{\partial x^{4}}+2 k \frac{\partial^{4} \omega}{\partial x^{2} \partial y^{2}}+\frac{\partial^{4} \omega}{\partial y^{4}}=0
$$

The case $\mathbf{k}=0$ is of some importance for such plates, [3]. If we wanted to solve the resulting equation $(k=0)$ in $x>0, y>0$ with the plate clamped along $x=0$ and having discontinuous boundary conditions along $\mathrm{y}=0$, we would quickly arrive at equations (1.1). For, in such a situation (with $k=0$ ), we seek solution $\omega(x, y)$ in the form

$$
\omega(x, y)=\int_{0}^{\infty} \frac{f(\lambda)}{\lambda}\left[e^{-\lambda y / \sqrt{2}} \sin \frac{\lambda y}{\sqrt{2}}\right]\left[e^{-\lambda x}-\cos \lambda x+\sin \lambda x\right] d \lambda,
$$

where $f(\lambda)$ is to be determined. This form is seen to satisfy the Partial Differential Equation, the condition of clampness along $x=0$ (i.e. $\omega=\frac{\partial \omega}{\partial x}=0$, on $x=0, y>0$ ) and the condition that $\omega=0$ on $y=0$ in $x>0$. If now, the plate is bent by bending moments of magnitude $\ln (x)$ in $0<x<1 \quad($ on $=0)$ and is clamped along $x>1$, (on $y=0)$, then $f(\lambda)$ must satisfy

$$
\int_{0}^{\infty} \lambda f(\lambda)\left(e^{-\lambda x}-\cos \lambda x+\sin \lambda x\right) d x=\mu \ln (x) \quad \text { in } 0<x<1
$$

and

$$
\int_{0}^{\infty} f(\lambda)\left(e^{-\lambda x}-\cos \lambda x+\sin \lambda x\right) d x=0 \quad \text { in } x>1
$$

where $\mu$ is an appropriate (material) constant, [3]. These equations are a particular case of equations(1.1). We propose to solve such equations in this note.

\section{PRELIMINARIES.}

We shall need the following known definitions and results. The results from the Mellin Transform theory can be found in Titchmarsh [4].

We define the Mellin Transform and inverse Mellin Transform, under appropriate conditions, respectively as:

$$
\begin{aligned}
\mathrm{M}[\mathrm{f}(\mathrm{x}) ; \mathrm{s}] & =\int_{0}^{\infty} \mathrm{f}(\mathrm{x}) \mathrm{x}^{s-1} \mathrm{dx} \\
& =\mathrm{f}^{*}(\mathrm{~s}), \text { where } \mathrm{s}=\sigma+\mathrm{i} \tau, \quad a<\sigma<\mathrm{b},-\infty<\tau<\infty, \text { and } \\
\mathrm{M}^{-1}\left[\mathrm{f}^{*}(\mathrm{~s}) ; \mathrm{x}\right] & =\frac{1}{\frac{\pi}{\mathrm{i}}} \int_{\sigma-\mathrm{i} \infty}^{\sigma+\mathrm{i} \infty} \mathrm{f}^{*}(\mathrm{~s}) \mathrm{x}^{-8} \mathrm{ds} \\
& =\mathrm{f}(\mathrm{x}) .
\end{aligned}
$$

Throughout this note, we shall denote the Mellin Transform of a function $f$ by $f^{*}$.

A function $k$ is said to be a Fourier Kernel when for

and

$$
\int_{0}^{\infty} k(x t) f(t) d t=g(x)
$$

$$
\int_{0}^{\infty} k(x t) g(t) d t=f(x)
$$


for some suitable functions $f \& g$. Furthermore, if $\mathbf{k}^{*}(\mathrm{~s})$ denotes the Mellin transform of $\mathbf{k}(\mathrm{x})$, then

$$
\mathbf{k}^{*}(\mathrm{~s}) \mathbf{k}^{*}(1-\mathrm{s})=1
$$

on some line of the complex s-plane.

The Erdelyi-Kober [5] operators are given by,

and

$$
\eta, \alpha(0, x ; \sigma) f=\frac{\sigma}{\Gamma(\alpha)} \bar{x}^{\sigma(\eta+\alpha)} \int_{0}^{x}\left(x^{\sigma-t \sigma}\right)^{\sigma+1} t^{\sigma(\eta+1)-1} f(t) d t, \alpha>0
$$

$$
\eta, \alpha(\mathrm{x}, \boldsymbol{\mathrm { x }}: \sigma) \mathrm{f}=\frac{\sigma}{\Gamma(\alpha)} \mathrm{x}^{\sigma \eta} \int_{\mathrm{x}}^{\infty}\left(\mathrm{t} \sigma-\mathrm{x}^{\sigma}\right)^{\alpha^{-1}} \mathrm{t}^{\sigma(1-\alpha-\eta)-1} \mathrm{f}(\mathrm{t}) \mathrm{dt}, \alpha>0
$$

It is an easy matter to see that

$$
\mathrm{M}\left[\mathrm{I}_{\eta, \alpha}(0, \mathrm{x}: \sigma) \mathrm{f}(\mathrm{x}): \mathrm{s}\right]=\frac{\Gamma(1+\eta-\mathrm{s} / \sigma)}{\Gamma(1+\eta+\alpha-\mathrm{s} / \sigma)} \mathrm{f}^{*}(\mathrm{~s})
$$

and

$$
\mathrm{M}\left[\mathrm{K}_{\eta, \alpha}(\mathrm{x}, \mathrm{\infty} ; \sigma) \mathrm{f}(\mathrm{x}) ; \mathrm{s}\right]=\frac{\Gamma(\eta+\mathrm{s} / \sigma)}{\Gamma(\eta+\alpha+\mathrm{s} / \sigma)} \mathrm{f}^{*}(\mathrm{~s}) .
$$

The inversion form of those equations give us the following two results.

$\begin{aligned} \text { Lemma 1. } \quad \mathbf{M}^{-1}\left[\frac{\Gamma\left(1+\eta-\frac{\mathrm{s}}{\sigma}\right)}{\Gamma\left(1+\eta+\alpha-\frac{\mathbf{s}}{\sigma}\right)} \mathrm{f}^{*}(\mathrm{~s}) ; \mathrm{x}\right] & ={ }_{\eta, \alpha}^{\mathrm{I}, \alpha}(0, \mathbf{x} ; \sigma) \mathbf{M}^{-1}\left[\mathrm{f}^{*}(\mathrm{~s}) ; \mathrm{x}\right] \\ & ={ }_{\eta, \alpha}^{\mathrm{I}, \alpha}(0, \mathbf{x} ; \sigma) \mathrm{f}\end{aligned}$

Lemma 2. $\quad \mathbf{M}^{-1}\left[\frac{\Gamma\left(\eta+\frac{s}{\sigma}\right)}{\Gamma\left(\eta+\alpha+\frac{s}{\sigma}\right)} \mathrm{f}^{*}(\mathrm{~s}) ; \mathrm{x}\right] \quad={ }_{\eta} \mathrm{K}, \alpha(\mathrm{x}, \boldsymbol{\infty} ; \sigma) \mathbf{M}^{-1}\left[\mathrm{f}^{*}(\mathrm{~s}) ; \mathrm{x}\right]$

$$
={ }_{\eta} \mathrm{K}, \alpha(\mathbf{x}, \boldsymbol{\infty} ; \sigma) \mathrm{f}
$$

Note that ${ }_{\eta, 0}^{\mathrm{I}}, 0{ }_{\eta} \mathrm{K}_{0}=\mathrm{I}$, the identity operator.

Now an important result from the theory of Mellin Transform.

Lemma 3. The Parseval Theorem, [4]

the convolution of $f$ and $g$.

$$
M^{-1}\left[f^{*}(s) g^{*}(s) ; x\right] \quad=\int_{0}^{\infty} f(x) g\left(\frac{x}{t}\right) \frac{1}{t} d t
$$

$$
=\text { fog, }
$$

Next we give a formal description of the method we shall employ in solving dual integral equations (1.1) for arbitrary functions $h_{1}$ and $h_{2}$. These dual equations (1.1), are equivalent to

$$
\begin{gathered}
\int_{0}^{t} m_{1}\left(\frac{t}{x}\right) \frac{1}{x} d x \int_{0}^{\infty} \phi(u) h_{1}(u x) d u=\psi_{1}(t), 0<t<1 \\
\int_{t}^{\infty} m_{2}\left(\frac{t}{x}\right) \frac{1}{x} d x \int_{0}^{\infty} \phi(u) h_{2}(u x) d u=\psi_{2}(t), \quad t>1 .
\end{gathered}
$$


Here the functions $\psi_{1}$ and $\psi_{2}$ are defined in terms of the functions $f$ and $g$ and (as yet) arbitrary functions $m_{1}$ and $m_{2}$ by the equations.

and

$$
\psi_{1}(t)=\int_{0}^{t} f(x) m_{1}\left(\frac{t}{x}\right) \frac{1}{x} d x
$$

$$
\psi_{2}(t)=\int_{t}^{\infty} g(x) m_{2}\left(\frac{t}{x}\right) \frac{1}{x} d x .
$$

Suppose now we find functions $m_{1}$ and $m_{2}$ such that

$$
\int_{1}^{\infty} m_{1}(y) h_{1}\left(\frac{z}{y}\right) \frac{1}{y} d y=\int_{0}^{1} m_{2}(y) h_{2}\left(\frac{z}{y}\right) \frac{1}{y} d y=k(z) \text {, say }
$$

then the unknown function $\phi$ is the solution of the single integral equation

$$
\int_{0}^{\infty} \phi(u) k(u t) d u=\psi(t)
$$

where

$$
\psi(t)=\psi_{1}(t) H(1-t)+\psi_{2}(t) H(t-1)
$$

$H(t)$ being the Heaviside function. To determine the arbitrary functions $m_{1}$ and $m_{2}$, we exploit the theory of Mellin Transforms.

Now, due to the result of lemma 3 , we can write the equation (2.4) as

$$
\mathrm{m}_{1}^{*}(\mathrm{~s}) \mathrm{h}_{1}^{*}(\mathrm{~s})=\mathrm{m}_{2}^{*}(\mathrm{~s}) \mathrm{h}_{2}^{*}(\mathrm{~s})=\mathrm{k}^{*}(\mathrm{~s})
$$

whence

$$
\frac{\mathrm{m}_{1}^{*}(\mathrm{~s})}{\mathrm{m}_{2}^{*}(\mathrm{~s})}=\frac{\mathrm{h}_{2}^{*}(\mathrm{~s})}{\mathrm{h}_{1}^{*}(\mathrm{~s})}
$$

It is then possible to decompose $\mathrm{h}_{2}^{*}(\mathrm{~s}) / \mathrm{h}_{1}^{*}(\mathrm{~s})$ in such a way so that $\mathrm{m}_{1}^{*}(\mathrm{~s})$ and $\mathrm{m}_{2}^{*}(\mathrm{~s})$ are appropriately determined and eventually the functions $\mathrm{m}_{1}$ and $\mathrm{m}_{2}$ and hence $\psi_{1}$ and $\psi_{2}$ are then known. Next, to find $\phi$, from (2.5), we have due to lemma 3,

$$
\begin{gathered}
\phi^{*}(1-s) k^{*}(s)=\psi^{*}(s), \\
\text { i.e., } \quad \phi^{*}(s)=\frac{\psi^{*}(1-s)}{k^{*}(1-s)}=\psi^{*}(1-s) h^{*}(s), \text { say }
\end{gathered}
$$

Then the last equation is equivalent to

$$
\phi(x)=\int_{0}^{\infty} \psi(t) h(x t) d t
$$

giving us the required solution of the system (1.1), where

$$
h(x)=M^{-1}\left[\frac{1}{k^{*}(1-s)} ; x\right] \text {. }
$$

\section{THE DUAL INTEGRAL EQUATIONS I.}

We consider now

$$
\begin{gathered}
\int_{0}^{\infty} \phi(t) h_{1}(x t) d t=f(x), 0<x<1 \\
\int_{0}^{\infty} \phi(t) h_{2}(x t) d t=g(x), x>1
\end{gathered}
$$

where 


$$
h_{1}(x)=e^{-x}-\cos x+\sin x
$$

and

$$
h_{2}(x)=x^{\nu}\left(e^{-x}-\cos x+\sin x\right), \quad-1 \leq \nu \leq 1,
$$

the functions $f$ and $g$ are known and $\phi$ is to be determined. Now, for an appropriate complex $s$, the Mellin transforms of $h_{1}$ and $h_{2}$ are, respectively, [6, Chapter 6],

$$
\begin{aligned}
\mathrm{h}_{1}^{*}(\mathrm{~s}) & =2 \sqrt{2} \Gamma(\mathrm{s}) \sin \frac{1}{4} \pi \mathrm{s} \sin \frac{1}{4} \pi(\mathrm{s}+1) \\
& =\pi^{\frac{1}{2}} 2^{2 \mathrm{~s}-1} \frac{\Gamma\left(\frac{1}{2}+\frac{\mathrm{s}}{4}\right) \Gamma\left(\frac{3}{4}+\frac{\mathrm{s}}{4}\right)}{\Gamma\left(1-\frac{\mathrm{s}}{4}\right) \Gamma\left(\frac{3}{4}-\frac{\mathrm{s}}{4}\right)} \\
\mathrm{h}_{2}^{*}(\mathrm{~s}) & =\mathrm{h}_{1}^{*}(\mathrm{~s}+\nu)
\end{aligned}
$$

Then

$$
\frac{\mathrm{m}_{2}^{*}(\mathrm{~s})}{\mathrm{m}_{1}^{*}(\mathrm{~s})}=\frac{\mathrm{h}_{1}^{*}(\mathrm{~s})}{\mathrm{h}_{2}^{*}(\mathrm{~s})}=\frac{\Gamma\left(\frac{1}{2}+\frac{\mathrm{s}}{4}\right) \Gamma\left(\frac{3}{4}+\frac{\mathrm{s}}{4}\right) \Gamma\left(1-\frac{\nu}{4}-\frac{\mathrm{s}}{4}\right) \Gamma\left(\frac{3}{4}-\frac{\nu}{4}-\frac{\mathrm{s}}{4}\right)}{2^{2 \nu} \Gamma\left(1-\frac{\mathrm{s}}{4}\right) \Gamma\left(\frac{3}{4}-\frac{\mathrm{s}}{4}\right) \Gamma\left(\frac{1}{2}+\frac{\nu}{4}+\frac{\mathrm{s}}{4}\right) \Gamma\left(\frac{3}{4}+\frac{\nu}{4}+\frac{\mathrm{s}}{4}\right)}
$$

whence,

and

$$
\mathrm{m}_{1}^{*}(\mathrm{~s})=2^{2 \nu} \frac{\Gamma\left(1-\frac{s}{4}\right) \Gamma\left(\frac{3}{4}-\frac{s}{4}\right)}{\Gamma\left(1-\frac{\nu}{4}-\frac{s}{4}\right) \Gamma\left(\frac{3}{4}-\frac{\nu}{4}-\frac{s}{4}\right)}
$$

From the equation (2.2),

$$
\mathrm{m}_{2}^{*}(\mathrm{~s})=\frac{\Gamma\left(\frac{1}{2}+\frac{\mathrm{s}}{4}\right) \Gamma\left(\frac{3}{4}+\frac{\mathrm{s}}{4}\right)}{\Gamma\left(\frac{1}{2}+\frac{\nu}{4}+\frac{\mathrm{s}}{4}\right) \Gamma\left(\frac{3}{4}+\frac{\nu}{4}+\frac{\mathrm{s}}{4}\right)}
$$

$$
\begin{aligned}
\psi_{1}(t) & =\int_{0}^{t} f(x) m_{1}\left(\frac{t}{x}\right) \frac{1}{x} d x \\
& =\int_{0}^{\infty} f(x) \bar{m}_{1}\left(\frac{t}{x}\right) \frac{1}{x} d x
\end{aligned}
$$

where $\bar{m}_{1}(x)=m_{1}(x) H(x-1), H$ being the Heaviside function. Due to lemma 3 , we have for $-1<\nu<0$,

by repeated use of lemma 1 .

$$
\begin{aligned}
& \psi_{1}(\mathrm{t})=\mathrm{M}^{-1}\left[\mathrm{~m}_{1}^{*}(\mathrm{~s}) \mathrm{f}^{*}(\mathrm{~s}) ; \mathrm{t}\right] \\
& =M^{-1}\left[2^{2 \nu} \frac{\Gamma\left(1-\frac{s}{4}\right) \Gamma\left(\frac{3}{4}-\frac{s}{4}\right)}{\Gamma\left(1-\frac{\nu}{4}-\frac{s}{4}\right) \Gamma\left(\frac{3}{4}-\frac{\nu}{4}-\frac{s}{4}\right)} f^{*}(s) ; t\right] \\
& =2^{2 \nu} I_{0,-\frac{\nu}{4}}(0, t: 4) \quad-\frac{1}{4},-\frac{\nu}{4}{ }^{(0, t ; 4) f,}
\end{aligned}
$$

But if $0<\nu<1$, then

$$
\psi_{1}(\mathrm{t})=\mathrm{M}^{-1}\left[2^{2 \nu} \frac{\Gamma\left(\frac{3}{4}-\frac{s}{4}\right) \Gamma\left(1-\frac{s}{4}\right)}{\Gamma\left(1-\frac{\nu}{4}-\frac{s}{4}\right) \Gamma\left(\frac{3}{4}-\frac{\nu}{4}-\frac{s}{4}\right)} \mathrm{f}^{*}(\mathrm{~s}) ; \mathrm{t}\right]
$$




$$
\begin{aligned}
& =2^{2 \nu} \quad I \frac{1}{4}, \frac{1}{4}-\frac{\nu}{4}(0, \mathrm{t} ; 4) \mathrm{M}^{-1}\left[\frac{\Gamma\left(1-\frac{\mathrm{s}}{4}\right)}{\Gamma\left(\frac{3}{4}-\frac{\nu}{4}-\frac{\mathrm{s}}{4}\right)} \mathrm{f}^{*}(\mathrm{~s}) ; \mathrm{t}\right] \\
& =2^{2 \nu-2} I \frac{1}{4}, \frac{1}{4}-\frac{\nu}{4}(0, t ; 4) M^{-1}\left[\frac{\Gamma\left(1-\frac{s}{4}\right)}{\Gamma\left(\frac{7}{4}-\frac{\nu}{4}-\frac{8}{4}\right)}(3-\nu-s) f^{*}(s) ; t\right]
\end{aligned}
$$

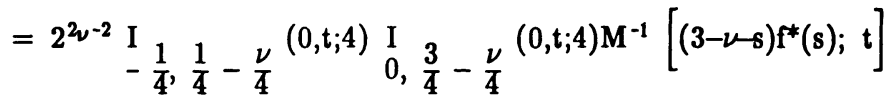

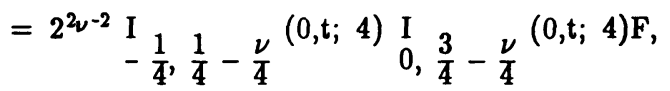

where $\quad F(x)=x^{\nu-2} \frac{d}{d x}\left[x^{3-\nu f(x)}\right]$.

Next, from equation (2.3) we have

$$
\begin{aligned}
\psi_{2}(t) & =\int_{t}^{\infty} g(x) m_{2}\left[\frac{t}{x}\right] \frac{1}{x} d x \\
& =\int_{0}^{\infty} g(x) \bar{m}_{2}\left[\frac{t}{x}\right] \frac{1}{x} d x,
\end{aligned}
$$

where $\overline{\mathrm{m}}_{2}(x)=\mathrm{m}_{2}(\mathrm{x}) \mathrm{H}(1-\mathrm{x})$. Then by lemmas 2 and 3 , for $0<\nu<1$,

$$
\begin{aligned}
& \psi_{2}(\mathrm{t}) \quad=\mathbf{M}^{-1}\left[\frac{\Gamma\left(\frac{1}{2}+\frac{s}{4}\right) \Gamma\left(\frac{3}{4}+\frac{s}{4}\right)}{\Gamma\left(\frac{1}{2}+\frac{\nu}{4}+\frac{s}{4}\right) \Gamma\left(\frac{3}{4}+\frac{\nu}{4}+\frac{s}{4}\right)} g^{*}(s) ; t\right] \\
& =\mathrm{K}_{\frac{1}{2}}, \frac{\nu}{4} \stackrel{(\mathrm{t}, \infty ; 4) \mathrm{K}}{\frac{3}{4},} \frac{\nu}{4}(\mathrm{t}, \infty ; 4) \mathrm{g}
\end{aligned}
$$

But if $-1<\nu<0$, then

$$
\begin{aligned}
& \psi_{2}(\mathrm{t})=\mathrm{K}_{\frac{1}{2}}, \frac{1}{4}+\frac{\nu}{4}(\mathrm{t}, \mathrm{w} ; 4) \mathrm{M}^{-1}\left[\frac{\Gamma\left(\frac{3}{4}+\frac{\mathrm{s}}{4}\right)}{\Gamma\left(\frac{1}{2}+\frac{\nu}{4}+\frac{\mathrm{s}}{4}\right)} \mathrm{g}^{*}(\mathrm{~s}) ; \mathrm{t}\right] \\
& =\frac{1}{4} \mathrm{~K}_{\frac{1}{2}}, \frac{1}{4}+\frac{\nu}{4}(\mathrm{t}, \infty ; 4) \mathrm{M}^{-1}\left[\frac{\Gamma\left(\frac{3}{4}+\frac{\mathrm{s}}{4}\right)}{\Gamma\left(\frac{3}{2}+\frac{\nu}{4}+\frac{\mathrm{s}}{4}\right)}(2+\nu+\mathrm{s}) \mathrm{g}^{*}(\mathrm{~s}) ; \mathrm{t}\right] \\
& =\frac{1}{4} \mathrm{~K}_{\frac{1}{2}}, \frac{1}{4}+\frac{\nu}{4}(\mathrm{t}, \infty ; 4) \mathrm{K}_{3}, \frac{3}{4}+\frac{\nu}{4}(\mathrm{t}, \infty ; 4) \mathrm{M}^{-1}\left[(2+\nu+\mathrm{s}) \mathrm{g}^{*}(\mathrm{~s}) ; \mathrm{t}\right] \\
& =\frac{1}{4} \mathrm{~K}_{\frac{1}{2}}, \frac{1}{4}+\frac{\nu}{4}(\mathrm{t}, \mathrm{w} ; 4) \mathrm{K}_{\frac{3}{4}}, \frac{3}{4}+\frac{\nu}{4}(\mathrm{t}, \mathrm{\infty} ; 4) \mathrm{G},
\end{aligned}
$$

where $G(x)=-x^{\nu+3} \frac{d}{d x}\left(x^{-\nu-2} g(x)\right)$.

Hence the function

$$
\psi(t)=\psi_{1}(t) H(1-t)+\psi_{2}(t) H(t-1)
$$

is completely known, with $\psi_{1}$ and $\psi_{2}$ as defined above by the equations (3.2) and (3.3). Further, from (2.7), 
Then, [7 Sec. 20.5],

$$
\begin{aligned}
\mathbf{k}^{*}(\mathrm{~s}) & =\mathrm{m}_{1}^{*}(\mathrm{~s}) \mathrm{h}_{1}^{*}(\mathrm{~s})=\mathrm{m}_{2}^{*}(\mathrm{~s}) \mathrm{h}_{2}^{*}(\mathrm{~s}) \\
& =\pi^{\frac{1}{2}} 2^{2 \mathrm{~s}+2 \nu-1} \frac{\Gamma\left(\frac{1}{2}+\frac{\mathrm{s}}{4}\right) \Gamma\left(\frac{3}{4}+\frac{\mathrm{s}}{4}\right)}{\Gamma\left(1-\frac{\nu}{4}-\frac{\mathrm{s}}{4}\right) \Gamma\left(\frac{3}{4}-\frac{\nu}{4}-\frac{\mathrm{s}}{4}\right)}
\end{aligned}
$$

$$
\begin{aligned}
h(x) & =M^{-1}\left[\frac{1}{k^{*}(1-s)} ; x\right] \\
& =M^{-1}\left[\pi^{-\frac{1}{2}} 2^{2 s-2 \nu-1} \frac{\Gamma\left(\frac{3}{4}-\frac{\nu}{4}+\frac{s}{4}\right) \Gamma\left(\frac{1}{2}-\frac{\nu}{4}+\frac{s}{4}\right)}{\Gamma\left(\frac{3}{4}-\frac{s}{4}\right) \Gamma\left(1-\frac{s}{4}\right)} ; x\right] \\
& =\pi^{-\frac{1}{2}} 2^{1-2 \nu} G_{04}^{20}\left[\left(\frac{x}{4}\right)^{4} \mid \frac{3}{4}-\frac{\nu}{4}, \frac{1}{2}-\frac{\nu}{4}, \frac{1}{4}, 0\right] .
\end{aligned}
$$

Finally the solution of the dual equations (3.1), can now be written as

$$
\phi(\mathrm{u})=\int_{0}^{\infty} \psi(\mathrm{t}) \mathrm{h}(\mathrm{ut}) \mathrm{dt}, \quad-1<\nu<1 .
$$

Note that the solution is also valid for $\nu= \pm 1$.

Next we list two special cases, which are of some interest.

Let $\nu=1, \mathrm{~g}(\mathrm{x})=0$ and $\mathrm{f}(\mathrm{x})=\mathrm{x}^{\alpha}, \alpha>-2$. Then

$$
\psi_{2}(\mathrm{t})=0
$$

and from (3.2a),

where $\quad F(x)=\frac{1}{x} \frac{d}{d x}\left(x^{2+\alpha}\right)=(2+\alpha) x^{\alpha}$.

$$
\psi_{1}(\mathrm{t})=\mathrm{I}_{0, \frac{1}{2}}(0, \mathrm{t} ; 4) \mathrm{F}
$$

That is,

$$
\begin{aligned}
\psi_{1}(t) & =(2+\alpha) I_{0, \frac{1}{2}}(0, t ; 4) x^{\alpha} \\
& =2 \pi^{-\frac{1}{2}}(2+\alpha) t^{-2} \int_{0}^{t}\left(t^{4}-x^{4}\right)^{-\frac{1}{2}} x^{3+\alpha} d x \\
& =4 \frac{\Gamma\left(1+\frac{\alpha}{4}\right)}{\Gamma\left(\frac{1}{2}+\frac{\alpha}{4}\right)} x^{\alpha} .
\end{aligned}
$$

The solution of the integral equations

$$
\begin{aligned}
& \int_{0}^{\infty} \phi(t) h_{1}(x t) d t=x^{\alpha}, 0<x<1 \\
& \int_{0}^{\infty} t \phi(t) h_{2}(x t) d t=0, x>1
\end{aligned}
$$

is then given by

where from (3.4),

$$
\phi(x)=4 \frac{\Gamma\left(1+\frac{\alpha}{4}\right)}{\Gamma\left(\frac{1}{2}+\frac{\alpha}{4}\right)} \int_{0}^{1} h(x t) t^{\alpha} d t
$$

$$
h(x)=\frac{1}{2} \pi^{-\frac{1}{2}} G_{04}^{20}\left[\left(\frac{x}{4}\right)^{4} \mid \frac{1}{2}, \frac{1}{4}, \frac{1}{4}, 0\right] .
$$

Evaluating the above integral, we obtain, [7 Sec. 20.5], for $\alpha>-2$, 


$$
\phi(\mathrm{x})=\frac{\Gamma\left(1+\frac{\alpha}{4}\right)}{\Gamma\left(\frac{1}{2}+\frac{\alpha}{4}\right)} \mathrm{G}_{15}^{21}\left[\left(\frac{\mathrm{x}}{4}\right)^{4} \mid \begin{array}{c}
\frac{3}{4}-\frac{\alpha}{4} \\
\frac{1}{2}, \frac{1}{4}, \frac{1}{4}, 0,-\frac{1}{4}-\frac{\alpha}{4}
\end{array}\right]
$$

as the solution of the system (3.5).

Let $\nu=-1, \mathrm{f}(\mathrm{x})=0$ and $\mathrm{g}(\mathrm{x})=\mathrm{x}^{\beta}, \beta<-\frac{1}{2}$.

Then

$$
\psi_{1}(\mathrm{t})=0
$$

and from (3.3a),

where

$$
\psi_{2}(\mathrm{t})=\frac{1}{4} \mathrm{~K}_{\frac{3}{4}, \frac{1}{2}}(\mathrm{t}, \infty ; 4) \mathrm{G},
$$

$$
\begin{aligned}
G(x) & =-x^{2} \frac{d}{d x}\left(x^{\beta-1}\right) \\
& =(1-\beta) x^{\beta}
\end{aligned}
$$

Therefore,

$$
\begin{aligned}
\psi_{2}(\mathrm{t}) & =\frac{1}{4}(1-\beta) \mathrm{K}_{\frac{3}{4}, \frac{1}{2}}(\mathrm{t}, \infty: 4) x^{\beta} \\
& =\pi^{-\frac{1}{2}}(1-\beta) t^{3} \int_{t}^{\infty}\left(x^{4}-t^{4}\right)^{-\frac{t}{2}} x^{\beta-2} d x \\
& =2 \frac{\Gamma\left(\frac{3}{4}-\frac{\beta}{4}\right)}{\Gamma\left(\frac{1}{4}-\frac{\beta}{4}\right)} t^{\beta} .
\end{aligned}
$$

Hence the solution of dual equation

$$
\begin{gathered}
\int_{0}^{\infty} \phi(t) h_{1}(x t) d t=0, \quad 0<x<1 \\
\int_{0}^{\infty}(x t)^{-1} \phi(t) h_{1}(x t) d t=x^{\beta}, \quad 1<x<\infty
\end{gathered}
$$

is given by

$$
\phi(x)=2 \frac{\Gamma\left(\frac{3}{4}-\frac{\beta}{4}\right)}{\Gamma\left(\frac{1}{4}-\frac{\beta}{4}\right)} \int_{1}^{\infty} h(x t) t \beta d t .
$$

Now from (3.4),

$$
h(x)=8 \pi^{-\frac{1}{2}} G_{04}^{20}\left[\left(\frac{x}{4}\right)^{4} \mid 1, \frac{3}{4}, \frac{1}{4}, 0\right],
$$

and on evaluating the above integral, we obtain,

$$
\phi(x)=16 \pi^{-\frac{1}{2}} \frac{\Gamma\left(\frac{3}{4}-\frac{\beta}{4}\right)}{\Gamma\left(\frac{1}{4}-\frac{\beta}{4}\right)} G_{15}^{30}\left[\left.\left(\frac{x}{4}\right)^{4}\right|_{1, \frac{3}{4},-\frac{1}{4}-\frac{\beta}{4}, \frac{1}{4}, 0} ^{\frac{3}{4}-\frac{\beta}{4}}\right], \quad \beta<-\frac{1}{2},
$$

as the solution of the system (3.6).

4. DUAL INTEGRAL EQUATIONS II.

Next we consider the system (1.1) with

$$
h_{1}(x)=e^{x}+\cos x-\sin x
$$


Now, [6],

$$
h_{2}(x)=x^{\nu} h_{1}(x),-1 \leq \nu \leq 1 .
$$

$$
\begin{aligned}
\mathrm{h}_{1}^{*}(\mathrm{~s}) & =2 \sqrt{2} \Gamma(\mathrm{s}) \sin \frac{1}{4} \pi(2+\mathrm{s}) \sin \frac{1}{4} \pi(1-\mathrm{s}) \\
& =\pi^{\frac{1}{2}} 2^{2 s-5} 2 \frac{\Gamma\left(\frac{3}{4}\right) \Gamma\left(\frac{1}{4}+\frac{8}{4}\right)}{\Gamma\left(\frac{1}{4}-\frac{8}{4}\right) \Gamma\left(\frac{1}{2}-\frac{8}{4}\right)}
\end{aligned}
$$

and $h_{2}^{*}(s)=h_{1}^{*}(s+\nu)$.

The equation (2.7) is satisfied, if we set

and

$$
\begin{gathered}
\mathrm{m}_{1}^{*}(\mathrm{~s})=2^{2 \nu} \frac{\Gamma\left(\frac{1}{4}-\frac{s}{4}\right) \Gamma\left(\frac{1}{2}-\frac{s}{4}\right)}{\Gamma\left(\frac{1}{4}-\frac{\nu}{4}-\frac{s}{4}\right) \Gamma\left(\frac{1}{2}-\frac{\nu}{4}-\frac{s}{4}\right)}, \\
\mathrm{m}_{2}^{*}(\mathrm{~s})=\frac{\Gamma\left(\frac{s}{4}\right) \Gamma\left(\frac{1}{4}+\frac{s}{4}\right)}{\Gamma\left(\frac{\nu}{4}+\frac{s}{4}\right) \Gamma\left(\frac{1}{4}+\frac{\nu}{4}+\frac{s}{4}\right)}
\end{gathered}
$$

$\mathbf{k}^{*}(s)=\sqrt{\pi} 2^{2 s+2 \nu-5} 2 \frac{\Gamma\left(\frac{s}{4}\right) \Gamma\left(\frac{1}{4}+\frac{s}{4}\right)}{\Gamma\left(\frac{\nu}{4}-\frac{s}{4}\right) \Gamma\left(\frac{1}{4}-\frac{\nu}{4}-\frac{s}{4}\right)}$.

Let $0<\nu<1$, then as before

$$
\begin{aligned}
\psi_{2}(t) & =\int_{t}^{\infty} g(x) m_{2}\left(\frac{t}{x}\right) \frac{1}{x} d x \\
& =M^{-1}\left[m_{2}^{*}(s) g^{*}(s) ; t\right] \\
& =M^{-1}\left[\frac{\Gamma\left(\frac{1}{4}+\frac{s}{4}\right) \Gamma\left(\frac{s}{4}\right)}{\Gamma\left(\frac{1}{4}+\frac{\nu}{4}+\frac{s}{4}\right) \Gamma\left(\frac{1}{2} \frac{\nu}{4}+\frac{s}{4}\right)} g^{*}(s) ; t\right] \\
& =K_{\frac{1}{4}, \frac{\nu}{4}}(t, \infty ; 4) K_{0, \frac{\nu}{4}}^{(t, \infty ; 4) g}
\end{aligned}
$$

Also,

$$
\begin{aligned}
\psi_{1}(t) & =\int_{0}^{t} f(x) m_{1}\left(\frac{t}{x}\right) \frac{1}{x} d x \\
& =M^{-1}\left[m_{1}^{*}(s) f^{*}(s) ; t\right] \\
& =M^{-1}\left[2^{2 \nu-2} \frac{\Gamma\left(\frac{1}{4}-\frac{s}{4}\right) \Gamma\left(\frac{1}{2}-\frac{s}{4}\right)}{\Gamma\left(\frac{1}{2}-\frac{\nu}{4}-\frac{8}{4}\right) \Gamma\left(\frac{5}{4}-\frac{\nu}{4}-\frac{8}{4}\right)}(1-\nu-s) f^{*}(s) ; t\right] \\
& =I \frac{3}{4}, \frac{1}{4}-\frac{\nu}{4}(0, x ; 4) I-\frac{1}{2}, \frac{3}{4}-\frac{\nu}{4}(0, x: 4) F
\end{aligned}
$$

where $F(x)=2^{2 \nu-2} x^{\nu} \frac{d}{d x}\left(x^{1-\nu f}(x)\right)$.

Now let $-1<\nu<0$. Then, as above

$$
\psi_{1}(\mathrm{t})=\mathrm{M}^{-1}\left[2^{2 \nu} \frac{\Gamma\left(\frac{1}{4}-\frac{s}{4}\right) \Gamma\left(\frac{1}{2}-\frac{8}{4}\right)}{\Gamma\left(\frac{1}{4}-\frac{\nu}{4}-\frac{8}{4}\right) \Gamma\left(\frac{1}{2}-\frac{\nu}{4}-\frac{8}{4}\right)} \mathrm{f}^{*}(\mathrm{~s}) ; \mathrm{t}\right]
$$




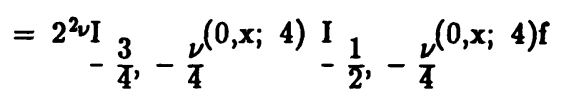

and

where $G(x)=-\frac{1}{4} x^{v+1} \frac{d}{d x}\left(x^{-v} g(x)\right)$.

$$
\begin{aligned}
\psi_{2}(\mathrm{t}) & =\mathrm{M}^{-1}\left[\frac{\Gamma\left(\frac{8}{4}\right) \Gamma\left(\frac{1}{4}+\frac{8}{4}\right)}{4 \Gamma\left(\frac{1}{4}+\frac{\nu}{4}+\frac{8}{4}\right) \Gamma\left(1+\frac{\nu}{4}+\frac{8}{4}\right)}(\nu+8) \mathrm{g}^{*}(\mathrm{~s}) ; \mathrm{t}\right] \\
& =\mathrm{K}_{0, \frac{1}{4}+\frac{\nu}{4}(\mathrm{x}, \infty, 4) \mathrm{K}} \mathrm{K}_{\frac{1}{4}, \frac{3}{4}+\frac{\nu}{4}}(\mathrm{x}, \infty ; 4) \mathrm{G},
\end{aligned}
$$

Hence the solution of the system is given by

where

$$
\phi(x)=\int_{0}^{\infty} \psi(t) h(x t) d t,
$$

and

$$
\begin{gathered}
\psi(t)=\psi_{1}(t) H(1-t)+\psi_{2}(t) H(t-1), \\
h(x)=M^{-1}\left[\frac{1}{k^{*}}(1-s)^{;} x\right] \\
=\pi^{-\frac{1}{2}} 2^{\frac{9}{2}-2 \nu} G_{0,}^{20}\left[\left(\frac{x}{4}\right)^{4} \mid-\frac{\nu 1}{4}, \frac{1}{4}-\frac{\nu 3}{4}, \frac{1}{4}, \frac{1}{2}\right] .
\end{gathered}
$$

Special cases when $\nu= \pm 1$ can easily be derived from the general solution.

We also note that the solution of equations (1.1) where

and

$$
h_{1}(x)=\sqrt{x}\left(Y_{0}(x) \pm \frac{2}{\pi} K_{0}(x)\right)
$$

$$
h_{2}(x)=x^{\nu h_{1}}(x), \quad-1 \leq \nu \leq 1
$$

can also be obtained along similar lines. It is well known that the functions $h_{1}(x)$ are Fourier Kernels, [2].

This research is partially supported by grants from Natural Sciences and Engineering Research Council of Canada.

\section{REFERENCES}

1. Nasim, C; Sneddon; I.N. A General Procedure for Deriving Solutions of Dual Integral Equations. J. Engg. Math. Vol. 12, No. 2, April 1978, 115-128.

2. Aggarwala, B.D; Nasim, C. Solutions of an Ordinary Differential Equation as a Class of Fourier Kernels, Internat. J. Math. \& Math Sci. Vol. 13, No. 2, (1990), $397-404$.

3. Timo Shenko, S. Theory of Plates and Shells, McGraw Hill, New York, 1989.

4. Titchmarsh, E.C. Introduction to the Theory of Fourier Integrals, second edition, Oxford University Press. Oxford, 1966.

5. Erdelyi, A. Some Dual Integral Equations, SIAM J. of Appl. Math, Vol. 16 (1968), 1338-1340.

6. Erdelyi, A. et al. Tables of Integral Transforms Vol I \& II, McGraw Hill, New York, 1954.

7. Erdelyi, A. et al. Tables of Integral Transforms, Vol II., McGraw Hill, New York, 1954 . 


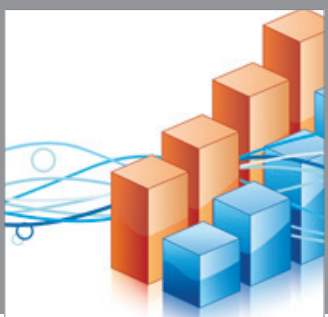

Advances in

Operations Research

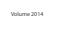

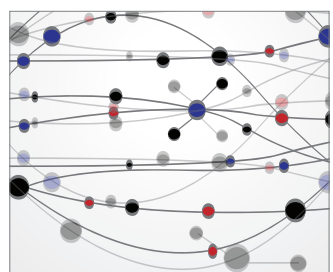

\section{The Scientific} World Journal
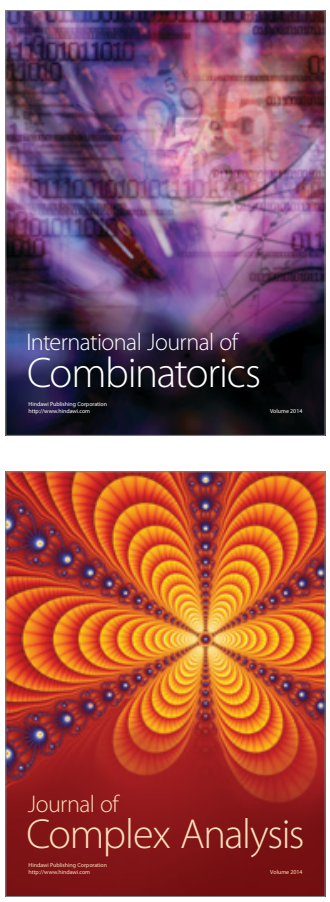

International Journal of

Mathematics and

Mathematical

Sciences
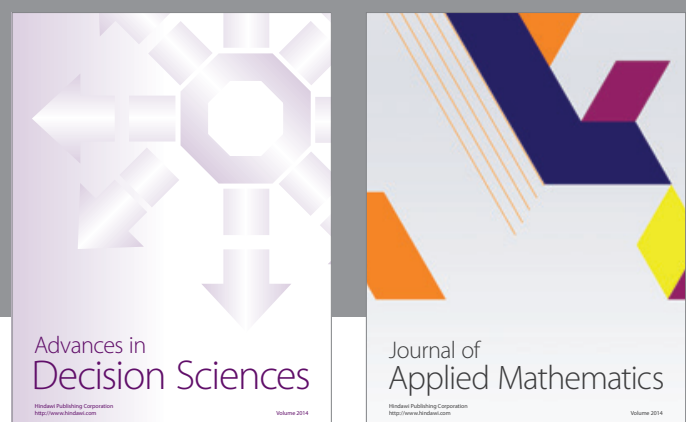

Journal of

Applied Mathematics
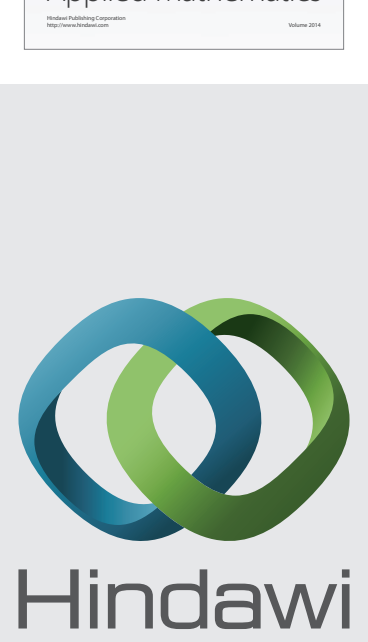

Submit your manuscripts at http://www.hindawi.com
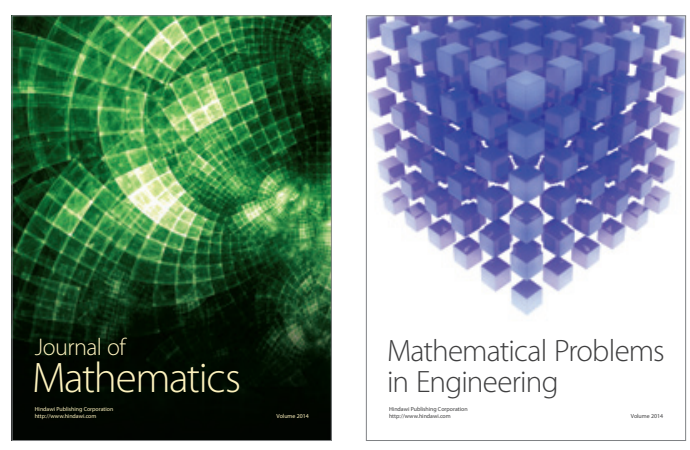

Mathematical Problems in Engineering
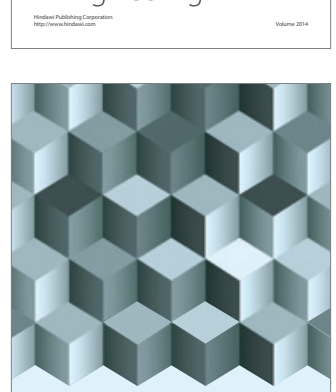

Journal of

Function Spaces
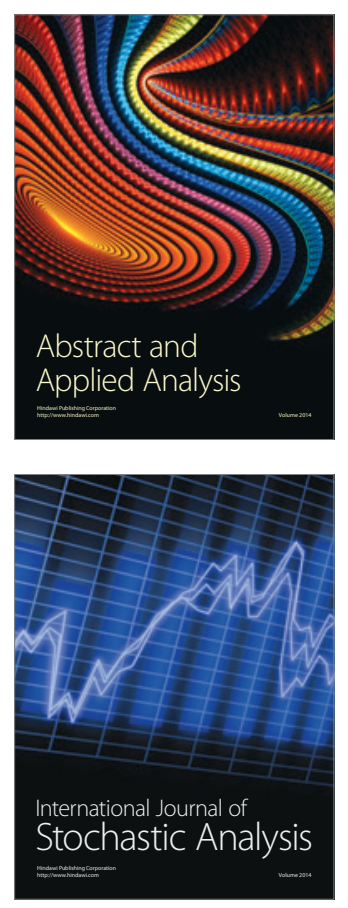

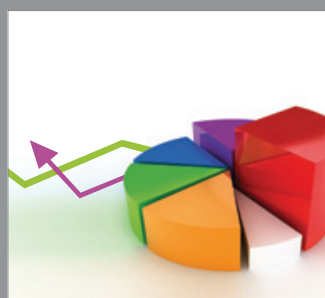

ournal of

Probability and Statistics

Promensencen
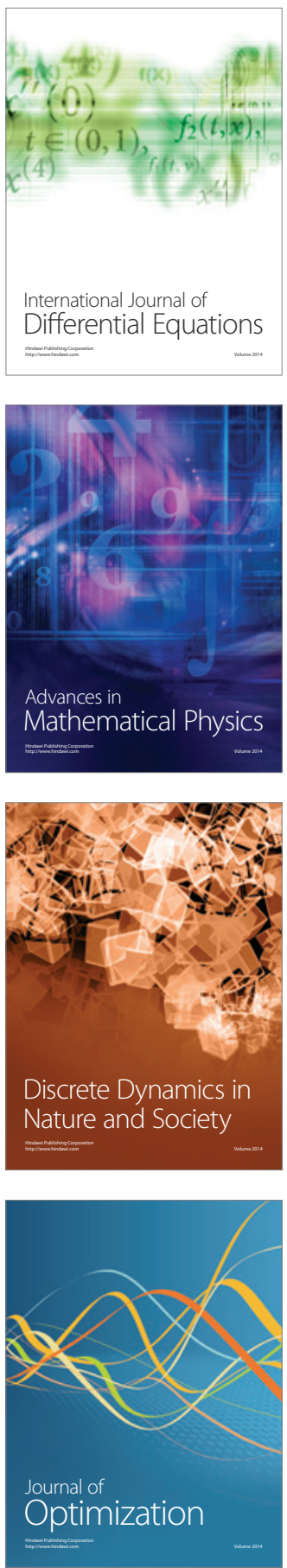